\title{
CNKSR2-related neurodevelopmental and epilepsy disorder: a cohort of 13 new families and literature review indicating a predominance of loss of function pathogenic variants
}

Leigh Ann Higa ${ }^{1,6}$, Jennifer Wardley², Christopher Wardley², Susan Singh ${ }^{3}$, Timothy Foster ${ }^{4}$ and Joseph J. Shen ${ }^{5,6^{*}}$ (1)

\begin{abstract}
Background: Pathogenic variants in connector enhancer of kinase suppressor of Ras-2 (CNKSR2) located on the $X$ chromosome (Xp22.12) lead to a disorder characterized by developmental delay and a characteristic seizure phenotype. To date, 20 affected males representing 13 different pathogenic variants have been published.

Case presentation: We identified an 8-year-old male with seizures, abnormal electroencephalogram (EEG) with epileptiform abnormalities in the right hemisphere, and developmental delay with notable loss of speech following seizure onset. Additional concerns include multiple nighttime awakenings, hyperactivity, and autism spectrum disorder. Genetic testing identified a de novo pathogenic nonsense variant in CNKSR2.

Through an active family support group, an additional 12 males are described, each harboring a different CNKSR2 variant. The clinical presentation and natural history consistently show early developmental delay, sleep disturbances, and seizure onset in childhood that is initially intractable but later becomes better controlled. Virtually all of the pathogenic variants are predicted to be loss of function, including genomic deletions, nonsense variants, splice site mutations, and small insertions or deletions.
\end{abstract}

Conclusions: This expanded knowledge, combined with functional studies and work with animal models currently underway, will enable a better understanding and improved ability to care for individuals with CNKSR2-related neurodevelopmental and epilepsy disorder.

Keywords: CNKSR2, CNK2, Epilepsy, ESES, CSWS, Developmental delay

*Correspondence: jojshen@ucdavis.edu

${ }^{5}$ Division of Genetics, Department of Pediatrics, UCSF Fresno, Fresno, CA, USA

Full list of author information is available at the end of the article

\begin{abstract}
Background
Connector enhancer of kinase suppressor of Ras-2 (CNKSR2, MIM 300724), with alternative designations in the literature including CNK2 and MAGUIN [18] was first described as Cnk in Drosophila melanogaster as a component of the Ras signaling pathway [16]. It is expressed predominantly in the brain ([12], Human Protein Atlas) and functions as a scaffold protein within
\end{abstract}


the postsynaptic density mediating numerous signaling pathways and neuronal morphogenesis, consistent with postulated roles in membrane and cytoskeletal remodeling $[3,4,8,10,11,19]$.

Since the initial characterization of a boy affected with a pathogenic variant in CNKSR2 by Houge et al. [7] (mental retardation, X-linked, Houge type (MRXSHG), MIM 301008), there have been a total of 20 affected males described in the literature, all with a consistent clinical phenotype of intellectual disability, developmental delay, and epilepsy $[1,2,5,6,15,17,20]$. Early developmental progress may be normal in rare instances, but global delays are observed consistently after the onset of seizures. Sleep disturbances are common as well. Seizures in CNKSR2-related neurodevelopmental and epilepsy disorder are typically difficult to control, and the electroencephalographic findings are fairly characteristic and categorized as encephalopathy with status epilepticus during slow wave sleep (ESES) as well as continuous spike waves in sleep (CSWS).

Approximately half of the known instances of CNKSR2-related disease are de novo in origin, and of the maternally transmitted cases, the majority of carrier mothers are neurologically without concerns. However, some females heterozygous for a CNKSR2 pathogenic variant have intellectual disability and seizures $[2,5,6$, $14,20]$.

A total of 13 different variants in these 20 males have been reported and, interestingly, all are expected to be loss of function, whether from genomic deletions encompassing the entire CNKSR2 locus, nonsense variants, intronic variants resulting in aberrant splicing, or small insertions/deletions predicted to lead to a frameshift and downstream premature protein truncation.

We diagnosed a child with CNKSR2-related neurodevelopmental and epilepsy disorder, and in conjunction with a family-driven effort through the international CNKSR2 Family Support Group also present a cohort of an additional 12 affected males from different families each harboring unique and previously undescribed pathogenic variants. The Case Report section will describe the proband as family 1 , and clinical descriptions of families 2 through 13 will be in Additional file 1 . The parents or legal guardians contributed the clinical and molecular genetic information and provided written informed consent for research participation in this study approved by the Institutional Review Board at Community Regional Medical Center. All individuals presented with seizures and, with the exception of one child (family 5), exhibited intellectual disability/developmental delay prior to seizure onset.

\section{Case presentation}

The proband for family 1 is an 8 -year-old male born at term weighing $3912 \mathrm{~g}$ after an unremarkable pregnancy and delivery. Family medical history is without close relatives with similar concerns and the parents deny consanguinity. Early developmental milestones include sitting at 7 months and walking at 17 months. At 2.5 years of age he spoke a few words, but then after the onset of seizures at age 4 , he became non-verbal. Slowly over the course of the intervening years he has regained some language and currently says about 10 words. Fine motor skills include operating a tablet computer and at least drawing a circle. He has been diagnosed with autism spectrum disorder. His first seizure was at 4 years of age with a prolonged staring episode. The next seizure occurred several months later but then became more frequent and harder to control. Multiple electroencephalograms (EEGs) have been read as epileptiform abnormalities in the right hemisphere, patterns consistent with LandauKleffner, and spiking occurring at night. He had been started on antiepileptic treatment (levetiracetam) without success, and now is somewhat better controlled on cannabidiol (CBD) oil with continued titration of the CBD:tetrahydrocannabinol (THC) formulation. Sleep is significantly disrupted with him generally being a light sleeper and having absence, tonic-clonic, and unusual laughing episodes (gelastic) seizures mainly at night. Melatonin has only slightly helped in the past, but now CBD oil may be of greater benefit. There are also nocturnal episodes of nonresponsiveness. Generally at baseline he is quite agitated and hyperactive. No dysmorphic features were noted on physical examination. Both head computed tomography $(\mathrm{CT})$ and brain magnetic resonance imaging (MRI) are without findings of note.

A diagnosis of CNKSR2-related neurodevelopmental and epilepsy disorder was made via genetic testing. Clinical whole exome sequencing (InVitae Corporation, San Francisco, CA, USA) identified a novel nonsense variant in CNKSR2, NC_000023.10: g.21627392T>G NM_014927.5: c.2349T>G, p.(Tyr783*). Subsequent testing of his mother determined that this CNKSR2 variant is not maternally inherited.

\section{Overview of cohort}

Our cohort includes 13 males from 13 families, with the case we diagnosed as family 1 . The age range is 3 years to 20 years, and the average age of our cohort is 8.7 years. Birth history consists of full term delivery for 11/13 males (85\%), and preterm delivery at 36 weeks and 36 weeks 6 days, for two males, respectively (15\%).

The two clinical characteristics observed universally in our cohort are seizures and intellectual disability (Table 1). The age of seizure onset ranges from 4 days 
Table 1 Clinical and molecular summary of CNKSR2-related neurodevelopmental and epilepsy disorder

\begin{tabular}{|c|c|c|c|c|c|c|}
\hline Family & $\begin{array}{l}\text { Age } \\
\text { (years) }\end{array}$ & $\begin{array}{l}\text { Age of } \\
\text { seizure onset }\end{array}$ & $\begin{array}{l}\text { Intellectual } \\
\text { disability? }\end{array}$ & $\begin{array}{l}\text { Maternal } \\
\text { origin? }\end{array}$ & Variant $^{a}$ & Source \\
\hline 1 & 8 & $4 y$ & $Y^{c}$ & $\mathrm{~N}$ & c. $2349 \mathrm{~T}>\mathrm{G}, \mathrm{p} .\left(\mathrm{Tyr} 783^{*}\right)$ & This study \\
\hline 2 & 21 & $15 \mathrm{~m}^{\mathrm{b}}$ & Y & Y & c. 1537 C > T, p.(Pro513Ser) ${ }^{e}$ & This study \\
\hline 3 & 5 & $2 y$ & $Y^{c}$ & $\mathrm{~N}$ & c.1988_1989del, p.(Arg663Asnfs*2) & This study \\
\hline 4 & 10 & $6 \mathrm{~m}$ & Y & $\mathrm{N}$ & c.1653_1656del, p.(Asn551Lysfs*4) & This study \\
\hline 5 & 9 & $4 y$ & $N \rightarrow Y^{d}$ & Y & c. 2545 C > T, p. $\left(\operatorname{Arg} 849^{*}\right)$ & This study \\
\hline 6 & 8 & $7 d$ & $Y^{c}$ & $\mathrm{~N}$ & $c .2145+1 G>A$ & This study \\
\hline 7 & 9 & $8 m$ & $Y^{c}$ & Y & arr [hg19] Xp22.12 $(21,278,397-21,678,707) \times 0$ & This study \\
\hline 8 & 7 & $2 y$ & Y & $\mathrm{N}$ & c. $1198 \mathrm{C}>\mathrm{T}$, p. $\left(\operatorname{Arg} 400^{*}\right)$ & This study \\
\hline 9 & 9 & $4 d$ & $Y^{c}$ & N & $c .2044+2 T>A$ & This study \\
\hline 10 & 5 & $2 y$ & $Y^{c}$ & $\mathrm{~N}$ & c.520-1G>A & This study \\
\hline 11 & 11 & $23 m$ & Y & $\mathrm{N}$ & c.2005del, p.(Ala669GInfs*48) & This study \\
\hline 12 & 3 & $2 y$ & $Y^{c}$ & $\mathrm{~N}$ & c. $1905-2 A>G$ & This study \\
\hline 13 & 8 & $2 y$ & $Y^{c}$ & N & c.2026_2027del, p.(Arg676Aspfs*2) & This study \\
\hline 14 & 7 & $2 y$ & Y & Y & arr [hg19] Xp22.12 $(21,322,029-21,678,137) \times 0$ & Aypar et al. [1] \\
\hline 15 & 5 & $2.5 y$ & Y & Y & arr [hg19] Xp22.12 $(21,375,312-21,609,484 \times 0$ & $\begin{array}{l}\text { Houge et al. [7], Vaags et al. } \\
\text { [17] pt } 3\end{array}$ \\
\hline \multirow[t]{2}{*}{16} & 6 & $2 y$ & $Y^{c}$ & Y & arr [hg19] Xp22.12 $(20,297,696-21,471,387) \times 0$ & Vaags et al. [17] pt 1 \\
\hline & 8 & $27 m$ & $Y^{c}$ & Y & & Vaags et al. [17] pt 2 \\
\hline \multirow[t]{2}{*}{17} & 12 & $8 d$ & $Y^{c}$ & Y & arr [hg19] Xp22.12 $(21,193,947-21,707,169) \times 0$ & Vaags et al. [17] pt 4 \\
\hline & 13 & N/A & $Y^{c}$ & Y & & Vaags et al. [17] pt 5 \\
\hline \multirow[t]{3}{*}{18} & 56 & $?^{\mathrm{b}}$ & $Y^{c}$ & Y & g.21458832_21458833ins, p.(Asp152Argfs*8) & $\begin{array}{l}\text { Vaags et al. [17] pt 6, Hu et al. } \\
\text { [8], family P180 }\end{array}$ \\
\hline & 58 & $?^{\mathrm{b}}$ & $Y^{c}$ & Y & & $\begin{array}{l}\text { Vaags et al. [17] pt 7, Hu et al. } \\
\text { [8], family P180 }\end{array}$ \\
\hline & 62 & $?^{\mathrm{b}}$ & $Y^{c}$ & Y & & $\begin{array}{l}\text { Vaags et al. [17] pt 8, Hu et al. } \\
\text { [8], family P180 }\end{array}$ \\
\hline \multirow[t]{3}{*}{19} & 18 & $3.5 y$ & $Y^{c}$ & Y & c. $2341 C>T, p .\left(\operatorname{Arg} 712^{*}\right)$ & Damiano et al. [5] \\
\hline & 12 & $3.5 y$ & Y & Y & & Damiano et al. [5] brother \\
\hline & $?$ & $3.5 y^{b}$ & Y & $?$ & & $\begin{array}{l}\text { Damiano et al. [5] maternal } \\
\text { uncle }^{f}\end{array}$ \\
\hline 20 & 9 & $2 y$ & Y & $\mathrm{N}$ & c. 2185 C > T, p. $\left(\operatorname{Arg} 729^{*}\right)$ & Sun et al. [15] \\
\hline 21 & 6 & $2 y$ & Y & $\mathrm{N}$ & c.2024_2027del, p.(Glu657Glyfs*41) & Bonardi et al. [2] pt 1 \\
\hline 22 & 21 & $3 y^{b}$ & $N \rightarrow Y^{d}$ & $\mathrm{~N}$ & c.246_247del, p.(Thr83Lysfs*30) & Bonardi et al. [2] pt 2 \\
\hline 23 & 12 & $4 y$ & $Y^{c}$ & $Y$ (mosaic) & c.457_461del, p.(Tyr153Serfs*5) & Bonardi et al. [2] pt 3 \\
\hline 24 & 10 & $3 y$ & $Y^{c}$ & $\mathrm{~N}$ & arr [hg19] Xp22.12 $(21,609,392-21,619,786) \times 0$ & Bonardi et al. [2] pt 5 \\
\hline \multirow[t]{2}{*}{25} & 5 & $4 y$ & Y & Y & arr [hg19] Xp22.12 $(21,606,698-21,616,207) \times 0$ & Daoqi et al. [6] \\
\hline & "Younger" & $3 y$ & Y & Y & & Daoqi et al. [6] brother \\
\hline 26 & 6 & N/A & Y & Y & c. $1904+1 G>A$ & Zhang et al. [20] \\
\hline \multicolumn{7}{|c|}{ Females } \\
\hline & - & N/A & $\mathrm{N}$ & $?$ & c.1537C > T, p.(Pro513Ser) & Mother in family 2 \\
\hline & - & N/A & $\mathrm{N}$ & $?$ & c.2545C > T, p. $\left(\operatorname{Arg} 849^{*}\right)$ & Mother in family 5 \\
\hline & - & N/A & $\mathrm{N}$ & $?$ & arr [hg19] Xp22.12 $(21,278,397-21,678,707) \times 1$ & Mother in family 7 \\
\hline & - & N/A & $\mathrm{N}$ & $?$ & arr [hg19] Xp22.12 $(21,322,029-21,678,137) \times 1$ & Mother in family 14 \\
\hline & - & N/A & $\mathrm{N}$ & $\mathrm{N}$ & arr [hg19] Xp22.12 $(21,375,312-21,609,484) \times 1$ & Mother in family 15 \\
\hline & - & N/A & $\mathrm{N}$ & $?$ & arr [hg19] Xp22.12 $(20,297,696-21,471,387) \times 1$ & Mother in family 16 \\
\hline & 16 & $6 y$ & Y & Y & c. $2341 C>T, p .\left(\operatorname{Arg} 712^{*}\right)$ & Sister in family 19 \\
\hline & $?$ & febrile & N & N & & Mother in family 19 \\
\hline 27 & 7 & $5 y$ & Y & $\mathrm{N}$ & c.2304G > A, (p.Trp768*) & Polla et al. [14] \\
\hline 28 & 41 & $6 y^{b}$ & $N \rightarrow Y^{d}$ & $?$ & arr [hg19] Xp22.12 $(21,523,673-21,558,329) \times 1$ & $\begin{array}{l}\text { Bonardi et al. [2], Lesca et al. } \\
\text { [9] pt } 4\end{array}$ \\
\hline
\end{tabular}


Table 1 (continued)

\begin{tabular}{|c|c|c|c|c|c|c|}
\hline Family & $\begin{array}{l}\text { Age } \\
\text { (years) }\end{array}$ & $\begin{array}{l}\text { Age of } \\
\text { seizure onset }\end{array}$ & $\begin{array}{l}\text { Intellectual } \\
\text { disability? }\end{array}$ & $\begin{array}{l}\text { Maternal } \\
\text { origin? }\end{array}$ & Variant $^{\mathrm{a}}$ & Source \\
\hline & $?$ & Junior high & Y & $\mathrm{N}$ & arr [hg19] Xp22.12 $(21,606,698-21,616,207) \times 1$ & Mother in family 25 \\
\hline & 33 & N/A & $?$ & $\mathrm{~N}$ & C. $1904+1 G>A$ & Mother in family 26 \\
\hline
\end{tabular}

$d$ Days, $m$ months, $y$ years, $N / A$ not applicable, $p t$ patient

${ }^{a}$ In this cohort, transcript NM_014927 (GRCh37p13) confirmed for families 1, 2, 4, 5, 6, 7, 8, 11, 12, 13

${ }^{\mathrm{b}}$ Seizures stopped in pre-teen years

c Minimal-to-no speech

d Developmental delay manifested after onset of seizures

e Classified by laboratory as variant of uncertain significance

${ }^{\mathrm{f}}$ Not tested for familial CNKSR2 pathogenic variant, but neurological and seizure phenotype matches that seen in the proband

of age to 4 years, with a mean age of 20.6 months. Seizure type is variable, and includes absence, atonic, tonic clonic, complex partial, focal, gelastic, dacrystic seizures. Face and hand twitching have also been reported. Six of 13 males (46.1\%) had EEG documenting electrical status epilepticus during sleep, also known as continuous spikes and waves during sleep (ESES/CSWS, families 2, 6, 8, 10, $11,13)$. Other EEG abnormalities reported include, but are not limited to, prolonged seizures, focal epilepsy, and clusters of seizures during day and night (Additional file 1). Four males were diagnosed with Landau Kleffner $(4 / 13$ or $30.8 \%$; family $7,8,10,13)$ and one has a diagnosis of Lennox Gastaut (1/13 or $7.7 \%$; family 6 ).

Additional neurodevelopmental diagnoses reported in more than one individual include autism $(8 / 13$ or $26.7 \%$, families $1,3,4,5,7,9,12,13)$ and attention deficit hyperactivity disorder $(3 / 13$ or $24 \%$; families $1,7,13)$. Further clinical information for the probands in families 2 through 13 are provided in Additional file 1.

Twelve (92.3\%) of the CNKSR2 variants in this cohort were classified as likely pathogenic or pathogenic, with the remaining one (missense variant in family 2 ) a variant of uncertain significance. As shown in Table 1, insertion/ deletion (in/del) variants and splicing variants were each seen in $4 / 13(30.8 \%)$ of affected individuals, nonsense variants were noted in three individuals (23.1\%), and one male harbored a contiguous deletion (7.7\%). All mothers completed genetic testing to establish carrier status. Three mothers were found to be carriers, for an incidence of $23.1 \%$, and none have had a history of seizures or neurodevelopmental concerns.

\section{Discussion and conclusions}

The neurodevelopmental phenotype and seizure types exhibited by the affected males in this cohort was consistent with and further validated the patterns and characteristics observed in the previously published cases of CNKSR2-related neurodevelopmental and epilepsy disorder (Table 1). The degree of developmental delay can be severe and disproportionately affect language skills, with 8 of the $13(62 \%)$ of the individuals in our cohort with very few or no words, similar to that described in the published individuals with at least 10 of 20 reported to have no or very minimal speech. Significant absence and generalized tonic-clonic seizures are observed, oftentimes both in the same individuals, which generally are difficult to control with anti-epileptic medication. Interestingly, with most if not all of the older affected individuals reported in the literature, despite the medically intractable nature of their epilepsy during early childhood, there appears to be resolution of the seizures and weaning off of anti-epileptic medication in the pre-teen years. The sole adult in our cohort (family 2) matched this natural history pattern with complete cessation of his seizures and no further medication by 10 years of age.

Additional characteristics were noted which expand our understanding of the neurobehavioral and clinical phenotype. Sleep disturbances were reported by the parents to be present in 8 of the $13(62 \%)$ affected males in this cohort, as first appreciated in Vaags et al. [17]. Significant hyperactivity was present in $6(46 \%)$ as reported by the parents. Four (31\%) of the affected males had significant feeding issues necessitating the use of a feeding tube in early childhood, but in later years there was not consistently observed failure to thrive in them or the others in the cohort (data not shown).

Another commonality extending from the published cases of CNKSR2-related neurodevelopmental and epilepsy disorder is the type of pathogenic variants detected-loss of function continues to be the predominant pathogenic molecular mechanism of disease. As shown in Table 1, in our cohort 3 nonsense variants, 1 genomic deletion encompassing the CNKSR2 locus, 4 small indels predicted to lead to a frameshift and downstream stop codon, and 4 splice site variants, have been identified; only family 2 harbored a missense variant classified as a variant of uncertain significance. This 
observation that virtually all pathogenic CNKSR2 variants are loss of function is consistent with this gene's probability of being loss-of-function intolerant (pLI) score of 1.0 and loss-of-function observed/expected upper bound fraction (LOEUF) score of 0.185 (gnomAD v2.1.1).

Of the 13 affected males in our cohort, ten represent de novo cases, while in three the variants are maternally inherited. Recent articles have indicated that females harboring heterozygous CNKSR2 pathogenic variants may have seizures, with some also manifesting neurodevelopmental concerns (family 26, [14]; family 27, [2]; mother and sister of family 19, [5]; mother of family 25 , [6]; mother of family 26, [20]). However, for another six mothers (three mothers in this cohort, family 14, [1]; family 15, [7]; family 16, [17], there were no reported seizures or intellectual disability. Skewed X-inactivation may be the underlying reason for this observed difference in penetrance $(85: 15$ in the mother of family 25 , [6] and 20:80 in family 26, [14], both with neurodevelopmental concerns, while reported to be $57: 43$ for the mother reported to have no neurological issues of family $15,[7]$ ).

The size of our cohort is one of the key strengths of this report, permits us to confirm and expand upon the previously reported clinical phenotype and, importantly, corroborates loss of function of CNKSR2 as the principal molecular pathogenic mechanism of disease. The main limitation in this cohort is the variable amount of information for each individual, due to the fact that the families are located on different continents and obtaining full clinical and molecular documentation is a logistical challenge. To address this, we have focused our clinical data collection on the key phenotypic features of developmental delay/intellectual disability, seizures, and sleep disturbance. Accordingly, we acknowledge that novel features of CNKSR2-related neurodevelopmental and epilepsy disorder may remain unrecognized at this time, and await further characterization.

In conclusion, we report on a cohort of affected individuals with CNKSR2-related neurodevelopmental and epilepsy disorder that doubles the number of known pathogenic variants and corroborates CNKSR2 loss of function as the predominant molecular mechanism of disease. The neurodevelopmental and clinical phenotype is consistent with that previously reported in the literature with prominent features of intellectual disability and seizures. Our large cohort further bolsters and extends previous observations including noting significant sleep disturbances, hyperactivity, and some early feeding concerns. Approximately half of all known females heterozygous for a pathogenic CNKSR2 variant have a similar but sometimes milder neurological phenotype, but the other half are without neurodevelopmental concerns or seizures. An under-recognized aspect of the epilepsy appears to be that it decreases in severity during childhood and, in almost all instances, resolves in the pre-teen years; knowing this natural history may be useful in guiding seizure management. Our hope is that understanding the complete phenotype of CNKSR2-related neurodevelopmental and epilepsy disorder will bring us closer towards the ultimate goal of investigating treatment options for individuals affected by this disease.

\section{Abbreviations}

CNKSR2: Connector enhancer of kinase suppressor of Ras-2; EEG: Electroencephalogram; ESES: Status epilepticus during slow wave sleep; CSWS: Continuous spike waves in sleep; EEG: Electroencephalogram; CBD: Cannabidiol; THC: Tetrahydrocannabinol; CT: Computed tomography; MRI: Magnetic resonance imaging; in/del: Insertion/deletion; pLl: Probability of being loss-of-function intolerant; LOEUF: Loss-of-function observed/expected upper bound fraction.

\section{Supplementary Information}

The online version contains supplementary material available at https://doi. org/10.1186/s12920-021-01033-7.

Additional file 1. Supplemental Clinical Information for Families 2 through 13

\section{Acknowledgements}

The authors would like to acknowledge the families of the CNKSR2 Family Support Group for their support and participation. The families of the CNKSR2 Family Support Group and the authors also would like to acknowledge the clinicians and genetic laboratories who contributed to the clinical and molecular diagnoses in this cohort.

\section{Authors' contributions}

SS, JW, and CW initiated and conceptualized this work and facilitated acquisition of clinical information and medical records in communication with families within the CNKSR2 Family Support Group. JS and TF clinically and molecularly evaluated family 1. LAH and JS viewed, interpreted and compiled accumulated medical records, drafted and revised the manuscript, which all co-authors reviewed and approved. All authors read and approved the final manuscript.

\section{Funding}

Not applicable.

\section{Availability of data and materials}

The sequence datasets generated during the current study are not publicly available because it is possible that individual privacy could be compromised. Data are available from the corresponding author upon reasonable request.

\section{Declaration}

\section{Ethics approval and consent to participate}

This research study was conducted in accordance with the Declaration of Helsinki, and approved by the Institutional Review Board (IRB) of Community Regional Medical Center (CRMC). Written informed consent for participation and all forms of personally identifiable data, including biochemical, clinical and biometric data was obtained for all patients; for patients under the age of 16 , written informed consent was obtained from their parents or legal guardians. 


\section{Consent for publication}

Written informed consent for publication of identifying images or other personal or clinical details was obtained from all of the participants. For participants under the age of 18 , written informed consent for publication of identifying images or other personal or clinical details was obtained from their parents or legal guardians.

\section{Competing interests}

The authors declare that they have no competing interests.

\section{Author details}

'Department of Pediatrics, Community Regional Medical Center, Fresno, CA, USA. ${ }^{2}$ CNKSR2 Family Support Group, Rochdale, Lancashire, UK. ${ }^{3}$ CNKSR2 Family Support Group, Sanger, CA, USA. ${ }^{4}$ Division of Pediatric Neurology, Department of Pediatrics, UCSF Fresno, Fresno, CA, USA. ${ }^{5}$ Division of Genetics, Department of Pediatrics, UCSF Fresno, Fresno, CA, USA. ${ }^{6}$ Division of Genomic Medicine, Department of Pediatrics, MIND Institute, University of California, Davis, 2825 50th Street, Sacramento, CA 95817, USA.

Received: 15 March 2021 Accepted: 1 July 2021

Published online: 15 July 2021

\section{References}

1. Aypar U, Wirrell EC, Hoppman NL. CNKSR2 deletions: a novel cause of X-linked intellectual disability and seizures. Am J Med Genet A. 2015;167(7):1668-70.

2. Bonardi CM, Mignot C, Serratosa JM, Giraldez BG, Moretti R, Rudolf G, et al. Expanding the clinical and EEG spectrum of CNKSR2-related encephalopathy with status epilepticus during slow sleep (ESES). Clin Neurophysiol. 2020;131(5):1030-9.

3. Bumeister R, Rosse C, Anselmo A, Camonis J, White MA. CNK2 couples NGF signal propagation to multiple regulatory cascades driving cell differentiation. Curr Biol. 2004;14(5):439-45.

4. Clapéron A, Therrien M. KSR and CNK: two scaffolds regulating RASmediated RAF activation. Oncogene. 2007;26(22):3143-58.

5. Damiano JA, Burgess R, Kivity S, Lerman-Sagie T, Afawi Z, Scheffer IE, et al. Frequency of CNKSR2 mutation in the X-linked epilepsy-aphasia spectrum. Epilepsia. 2017;58(3):e40-3.

6. Daoqi M, Guohong C, Yuan W, Zhixiao Y, Kaili X, Shiyue M. Exons deletion of CNKSR2 gene identified in X-linked syndromic intellectual disability. BMC Med Genet. 2020;21(1):69.

7. Houge G, Rasmussen IH, Hovland R. Loss-of-function CNKSR2 mutation is a likely cause of non-syndromic X-linked intellectual disability. Mol Syndromol. 2012;2(2):60-3.

8. Hu H, Haas SA, Chelly J, Van Esch H, Raynaud M, de Brouwer APM, et al. $X$-exome sequencing of 405 unresolved families identifies seven novel intellectual disability genes. Mol Psychiatry. 2016;21(1):133-48.
9. Lesca G, Rudolf G, Labalme A, Hirsch E, Arzimanoglou A, Genton P, et al. Epileptic encephalopathies of the Landau-Kleffner and continuous spike and waves during slow-wave sleep types: genomic dissection makes the link with autism: LKS and CSWSS: genomic links with Autism. Epilepsia. 2012;53(9):1526-38.

10. Lim J, Ritt DA, Zhou M, Morrison DK. The CNK2 scaffold interacts with vilse and modulates rac cycling during spine morphogenesis in hippocampal neurons. Curr Biol. 2014;24(7):786-92.

11. Liu L, Channavajhala PL, Rao VR, Moutsatsos I, Wu L, Zhang Y, et al. Proteomic characterization of the dynamic KSR-2 interactome, a signaling scaffold complex in MAPK pathway. Biochim Biophys Acta. 2009;1794(10):1485-95.

12. Nagase T, Ishikawa K, Suyama M, Kikuno R, Hirosawa M, Miyajima N, et al. Prediction of the coding sequences of unidentified human genes. XII. The complete sequences of 100 new cDNA clones from brain which code for large proteins in vitro. DNA Res. 1998;5(6):355-64.

13. Piton A, Redin C, Mandel J-L. XLID-causing mutations and associated genes challenged in light of data from large-scale human exome sequencing. Am J Hum Genet. 2013;93(2):368-83.

14. Polla DL, Saunders HR, de Vries BBA, van Bokhoven $\mathrm{H}$, de Brouwer APM. A de novo variant in the $X$-linked gene CNKSR2 is associated with seizures and mild intellectual disability in a female patient. Mol Genet Genomic Med. 2019;7(10):e00861.

15. Sun Y, Liu Y-D, Xu Z-F, Kong Q-X, Wang Y-L. CNKSR2 mutation causes the $X$-linked epilepsy-aphasia syndrome: a case report and review of literature. World J Clin Cases. 2018;6(12):570-6.

16. Therrien $\mathrm{M}$, Wong AM, Rubin GM. CNK, a RAF-binding multidomain protein required for RAS signaling. Cell. 1998;95(3):343-53.

17. Vaags AK, Bowdin S, Smith M-L, Gilbert-Dussardier B, Brocke-Holmefjord KS, Sinopoli K, et al. Absent CNKSR2 causes seizures and intellectual, attention, and language deficits. Ann Neurol. 2014;76(5):758-64.

18. Yao I, Hata Y, Ide N, Hirao K, Deguchi M, Nishioka H, et al. MAGUIN, a novel neuronal membrane-associated guanylate kinase-interacting protein. J Biol Chem. 1999;274(17):11889-96.

19. Zieger HL, Kunde S-A, Rademacher N, Schmerl B, Shoichet SA. Diseaseassociated synaptic scaffold protein CNK2 modulates PSD size and influences localisation of the regulatory kinase TNIK. Sci Rep. 2020;10(1):5709.

20. Zhang Y, Yu T, Li N, Wang J, Wang J, Ge Y, Yao R. Psychomotor development and attention problems caused by a splicing variant of CNKSR2. BMC Med Genomics. 2020;13(1):182.

\section{Publisher's Note}

Springer Nature remains neutral with regard to jurisdictional claims in published maps and institutional affiliations. 